\title{
Use of thermography as an auxiliary method to diagnose orofacial pain: a case study
}

Isadora Souza Spínola Faria ${ }^{1}$
https:///orcid.org/0000-0002-9888-550X
Renata Silva Amaral ${ }^{1}$
https:///orcid.org/0000-0002-9984-6027

Lylian Vieira de Paula ${ }^{1}$ https://orcid.org/0000-0001-9686-2585

Patrícia Vieira Salles ${ }^{1}$ https://orcid.org/0000-0002-7559-5235
Pontifícia Universidade Católica de Minas Gerais - PUC MG, Belo Horizonte, Minas Gerais, Brasil.

Conflict of interests: Nonexistent

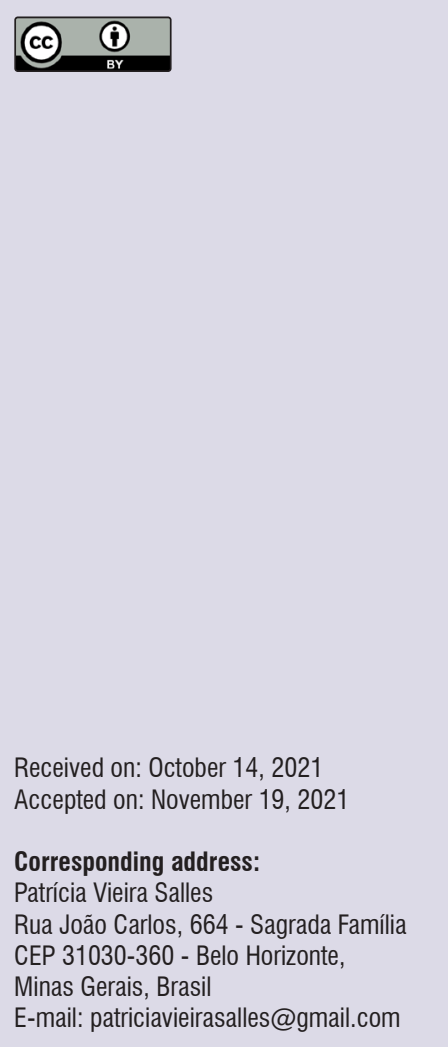

\section{ABSTRACT}

Thermography is an auxiliary diagnostic method widely used to assess soft tissues and thus diagnose neuropathic or inflammatory pain. The objective of this paper was to report a case in which thermography was used as an auxiliary method to diagnose neuropathic pain. The patient in question is a 43-year-old woman with complaints of pain and sensitivity in the upper and lower left dental arches, particularly when consuming alcoholic beverages. The case was assessed with thermography, followed by the Cold Stress Test. The symmetry of the thermographic images and the localized changes helped rule out neuropathic problems and diagnose the orofacial inflammatory pain. The thermography was used as an auxiliary instrument to diagnose orofacial pain, proving to be efficient. Also, the thermography helped rule out changes originating in peripheral, small-fiber, and inflammatory neuropathies, aiding the patient's differential diagnosis. The thermography also helped identify the thermo-anatomical points and, with the qualitative and quantitative analyses, noninvasively investigate the possible causes of the orofacial pain reported by the patient.

Keywords: Thermography; Facial Neuralgia; Facial Pain; Speech, Language and Hearing Sciences; Diagnosis 


\section{INTRODUCTION}

Pain is one of the main reasons why people seek healthcare. Besides being an important clinical symptom, it is a multidimensional sensation and experience, being therefore complex and most of the time subjective ${ }^{1}$.

Orofacial pain is any pain directly associated with soft and mineralized tissues, such as the skin, blood vessels, bones, teeth, glands, and muscles, present in the oral cavity and the face. It can also be reported in the head and neck region. The main causes of orofacial pain include odontogenic problems, headaches, neurogenic pathologies, musculoskeletal pain, psychogenic pain, cancer, infections, autoimmune phenomena, and tissue traumas ${ }^{1}$.

Temperature variations may occur in the human body due to various factors. They are directly related to the metabolic process and the sympathetic nervous system activity in the organs and systems - especially the vascular system, in which vasoconstriction may occur, decreasing the local temperature, or vasodilatation, which is normally related to lesions and inflammatory processes ${ }^{2}$.

Thermal analysis enables the professional to observe, based on temperature variations, the physiological changes that may occur in the human body. Thus, the type and site of the pain can be identified more precisely ${ }^{2}$.

Orofacial pain can be characterized as either acute or chronic pain. In the first case, when the patient complains of acute facial pain associated with quickly identifiable diseases (such as fractures, tumors, dental caries, sinus diseases, or infections), the diagnosis is not a problem, regardless of the therapeutic difficulties related to each case. On the other hand, patients who report persistent, diffuse, or chronic orofacial pain without evident abnormalities make up a population more difficult to diagnose and treat ${ }^{3}$.

In clinical practice, identifying neuropathic pain is difficult and rather complex. Abnormal findings in painful patients' neurological physical examination are suggestive of neuropathic pain. In the physical examination, the painful area is inspected and palpated. A precise assessment of the cranial nerves and muscle tone is also necessary, as well as, whenever possible, neuroimaging, such as cranial tomography and/or magnetic resonance imaging ${ }^{4}$.

In addition to these, thermography is a noninvasive painless diagnostic imaging method that can be performed by a speech-language-hearing therapist. It detects infrared radiation, produces and records images, showing the dynamics of the microcirculatory activity on the skin surface in real time. The thermal analysis encompasses the vascular, nervous, and musculoskeletal systems, which enables the assessment of inflammatory processes and endocrine, neurological, and oncological conditions ${ }^{5}$.

Thermography has been currently used in the assessment and documentation process of soft tissue pathologies, including peripheral nerves, nerve roots, tendons, muscles, and blood vessels, as well as joint and bone disorders. It provides additional information about some conditions (such as the myofascial pain) which cannot be identified with radiological or electroneuromyographic tests or the other laboratory examinations ${ }^{6}$.

Given the benefits and manifold applicability of thermography, the objective of this paper was to describe, with a case report, the use of thermography as an auxiliary method to diagnose neuropathic pain.

\section{CASE PRESENTATION}

This study was approved by the Research Ethics Committee of the Pontifícia Universidade Católica de Minas Gerais (Pontifical Catholic University of Minas Gerais), Brazil, under number 3.947.281 and CAAE 26117719.6.0000.5137. It is a case report of a 43-year-old woman who voluntarily agreed to participate in the research, signing the informed consent form. Her main complaint was orofacial pain and sensitivity.

The patient sought speech-language-hearing care reporting episodes of pain and sensitivity in the upper and lower left dental arches as her main complaint. The pain appeared after alcohol consumption, nocturnal teeth clenching, and migraines.

According to the patient, teeth clenching had been occurring for 3 years, whereas the sensitivity and pain following alcohol consumption had appeared 1 year before, with the symptoms occasionally disappearing.

She reported that her problem began as a toothache in both the upper and lower left arches. At the time of the assessment, she had symptoms on the left side of the face and occasionally scapular girdle pain on the left side.

When she came for speech-language-hearing care, she reportedly was not undergoing any medical follow-up. However, she had already had dental and acupuncture treatment in an attempt to solve her 
problem. She reported taking medications to ease the pain, which improved her symptoms.

In the craniocervical assessment, she reported back pain, the presence of nocturnal teeth clenching, and toothache in the upper and lower left molars. She also reported that the pain worsened daily when she woke up and in the evening.

Concerning the habits and episodes that caused and triggered the pain, the patient reported alcohol consumption, stress, and concern. In the assessment with the visual analog scale (VAS) ${ }^{7}$, she reported feeling two different "types" of pain. The first occurred when she clenched her teeth more tightly, being characterized by the patient as spontaneous pain, at degree 7 in the VAS. The second type of pain she mentioned occurred after consuming alcohol, appearing a few minutes after beginning the ingestion, always in the same region, characterized as very intense pain, at degree 9 in the VAS.

The patient sought professionals such as dentists, neurologists, acupuncturists, and speech-languagehearing therapists to be treated and ease the pain. According to her, she had already made some procedures, such as electrical stimulation, acupuncture, and medications, and she began doing physical exercises. During the dental treatment, she began using a myorelaxant occlusal splint and was submitted to a desensitizing treatment because, due to dental clenching, she had been having a gingival recession. The patient also had her dental radiographs taken, with normal results.

Hence, based on the patient's report, the first suspicion was of neurological change - as she reported pain triggered by innocuous stimuli, the radiography revealed no changes, and her pain was strong and paroxysmal.

Based on this information and the first impression, infrared thermography and the cold stress test were used to either confirm or rule out any neurological change, such as trigeminal neuralgia ${ }^{8}$.

The cold stress test was used mainly to confirm or rule out small-fiber neuropathy. This test also contributed to the differential diagnosis, as it analyzes the sympathetic neurovegetative system. To perform it, the patient must keep one of their hands immersed in water, whose temperature is between 10 and $20 \stackrel{\circ}{ } \mathrm{C}$ (degrees Celsius) ${ }^{9}$.

The patient's thermographic images were taken following the subject preparation protocol as instructed by the American Academy of Thermology ${ }^{10}$. The patient was acclimatized for 20 minutes. The images were taken with a thermographic camera (ThermoCAM ${ }^{\circledR}$ c2; FLIR ${ }^{\circledast}$ Systems, Inc., Wilsonville), fixed on a tripod, parallel to the face that would be photographed.

The room temperature was kept at $23 \stackrel{\circ}{\circ}$ (varying $1 \stackrel{\circ}{ } \mathrm{C}$ at the most) throughout the examination and the patient's minimum acclimatization time. The room temperature was monitored with a thermometer placed on a desk, within sight of the examiners. The air humidity in the examination setting was controlled and maintained between $45 \%$ and $50 \%$ relative humidity. The examination room was $12 \mathrm{~m}^{2}$, the windows were shut, and the light was fluorescent. The thermography camera was kept $70 \mathrm{~cm}$ from the patient's face.

The patient was instructed about the procedure and all her questions were answered during the medical history survey. To facilitate the acclimatization and imaging process, the patient was asked to keep her hair up and protected with a hair cap, wearing a strapless top, barefoot, with a disposable shoe cover. To ensure the same head position throughout the imaging process, a checkered poster measuring 1 meter wide by 1 and a half meters long with $10-\mathrm{cm}$ squares was placed on the wall behind the patient, controlling her shoulder position in relation to the squares.

The frontal images were taken respecting the $27^{\circ}$ to $37 \stackrel{\circ}{ } \mathrm{C}$ thermal window. Throughout the assessment, the patient was instructed not to touch or scratch her face, not occlude her teeth, and keep the masticatory and facial muscles relaxed.

Fourteen thermo-anatomical points, as proposed by Haddad $^{11}$, were analyzed separately and grouped into right and left hemiface, as shown in Figure 1.

For the cold stress test, the patient was instructed to put her left hand, which was wrapped in plastic, into a bowl with water at $15{ }^{\circ} \mathrm{C}$ for 5 minutes. Then, an image of her face was taken per minute. The water temperature was controlled with a cooking thermometer (digital cooking thermometer Espeto Alimentos Cozinha - Store 7D).

Hence, the TO was the acclimatization period (i.e., before beginning the cold stress test), $\mathrm{T} 1$ was the first minute of the test, and so on until T5 (Figure 2).

After taking the images in JPEG (Joint Photographic Experts Group), they were stored and analyzed with the Thermofy-Visionfy program, using the "thermoguided 1" color scale for the analysis and the thermoanatomical points from the research by Haddad $^{12}$ as a reference. The minimum (MIN T), mean (MEAN T), and maximum (MAX T) values were obtained, as well as the difference between the points on the left and right 
side and the mean total temperature of the right and left hemifaces.

The data were tabulated in a Microsoft Excel ${ }^{\circledR}$ database (Microsoft Corporation ${ }^{\circledR}$, Redmond, WA) for the quantitative analysis.

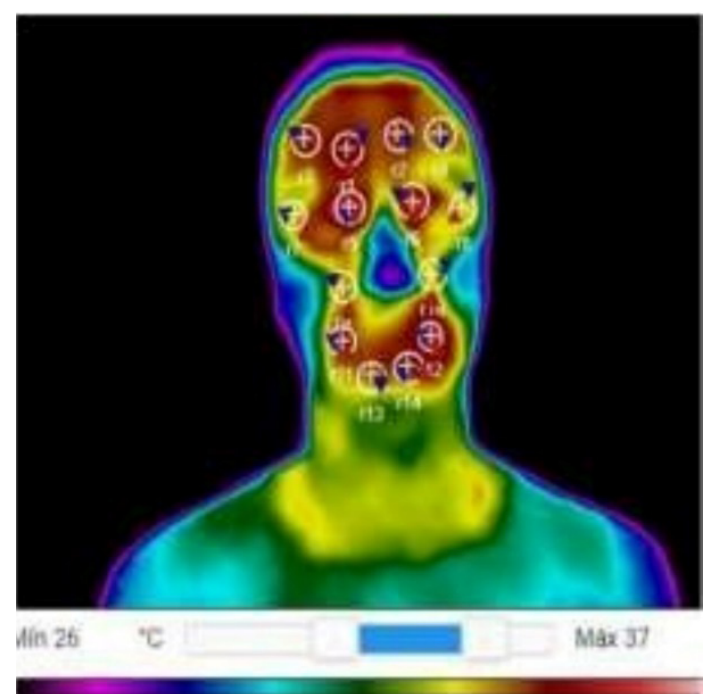

Figure 1. The patient's thermo-anatomical points at the beginning of the Cold Stress Test

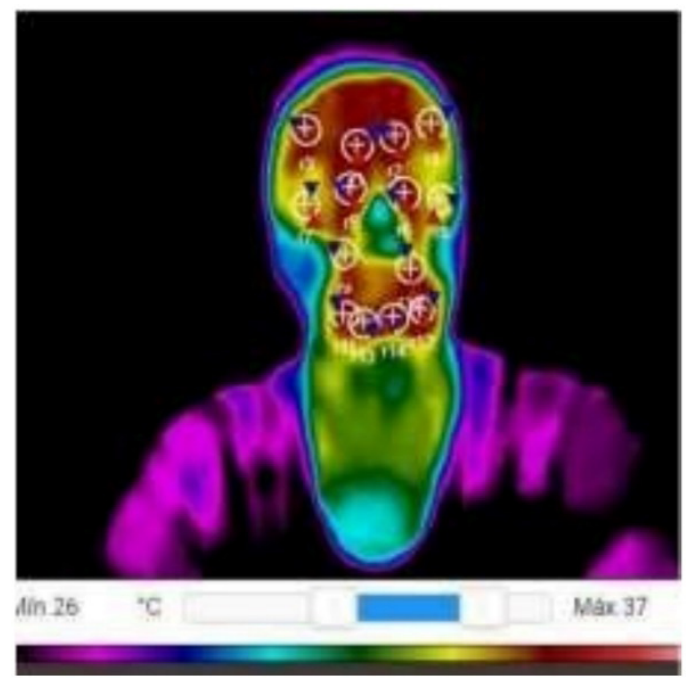

Figure 2. The patient's thermo-anatomical points at the fifth minute of the Cold Stress Test

\section{RESULTS}

Hence, thermography was used along with the cold stress test to either rule out or confirm the presence of neurological orofacial pain, such as small-fiber or inflammatory neuropathy. The patient's complaints led the researchers to believe that her condition had a peripheral origin, as she reported severe paroxysmal pain, according to the VAS, caused by innocuous stimuli.

The qualitative analysis of the patient's images taken during the cold stress test revealed localized changes in the thermographic images. This led to the researchers think that the condition had an inflammatory origin, such as in the cases of myofascial pain. If the changes had a neuropathic origin, the thermographic images would present regional patterns throughout the path of the facial nerves - which was not observed in the patient's images. After performing the cold stress test and perceiving the temperature symmetry at the end of the hands (Figure 3), the small-fiber neuropathies were ruled out.

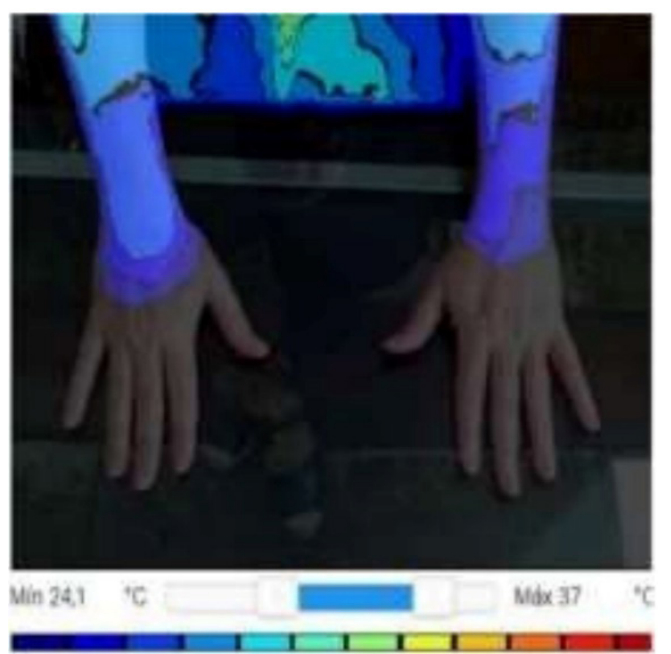

Figure 3. Image of the hands at the end of the cold stress test 
The qualitative analysis of the following thermographic images revealed their symmetric pattern. This helped rule out changes of neuropathic origin, which led the researchers to think of a localized change. Figure 4 showed a hyper-radiant thermographic point on the left side, where the patient reported episodes of pain and discomfort. The point in question was welldefined, and its temperature was higher than that of the patient's face, making it necessary to investigate a localized change on the lower left hemiface.

Lastly, also in the analyzed images, it is important to highlight the left frontal hemiface, where a hyper-radiant point is visible, though not symmetrical with the right hemiface. This finding can be related to the episodes of migraine reported by the patient.
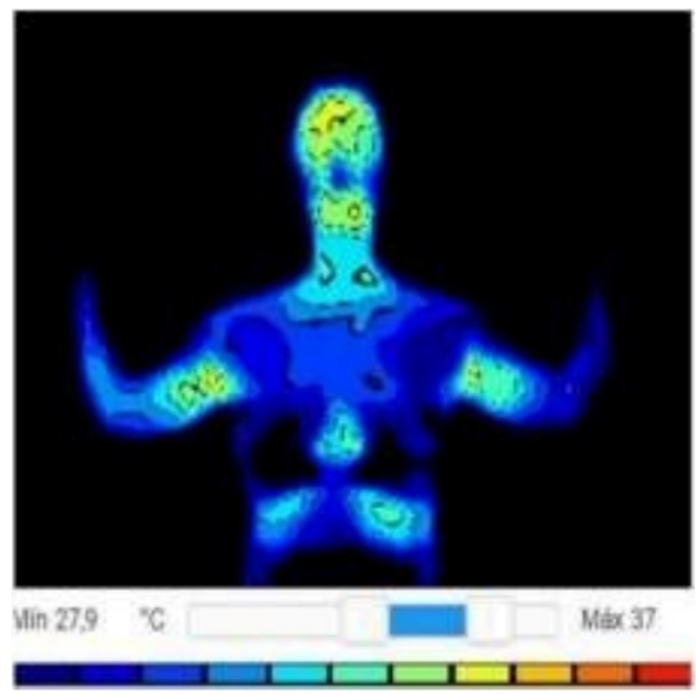

Figure 4. The patient's frontal image after performing the cold stress test. Attention is called to the thermographic point in the lower left hemiface and the hyper-radiant area in the upper right frontal

In addition to what was found with the cold stress test, the analysis of the sum of all thermo-anatomical points on the right and left hemifaces from T0 (acclimatization) to T5 (fifth minute of the cold stress test) revealed that the mean temperature variations ranged from $33.6 \stackrel{\circ}{\circ}$ to $34.7 \stackrel{\circ}{\circ}-$ i.e., a maximum variation of 1.1 으. The lowest mean temperature was the one found on the left hemiface in T1 (i.e., in the first minute of the cold stress test), while the highest temperature was the mean one found on the right hemiface in T3 of the cold stress test (Figure 5).

Analyses were made comparing the thermoanatomical points between the right and left hemiface in each analysis time. In T0, the temperature variations exceeded $0.3 \stackrel{\circ}{\circ}$ from the normal one in the following thermo-anatomical points: temporal, medial palpebral commissure, nasolabial, labial commissure, and labial ${ }^{12}$.

In $\mathrm{T} 1$ (the first minute of the cold stress test), the temperature variations exceeded $0.3 \stackrel{\circ}{\circ} \mathrm{C}$ in the following thermo-anatomical points: lateral palpebral commissures and nasolabial fold. In T2 and T3 they occurred in the nasolabial folds and labial commissures. In T4, they occurred in the temporal points, lateral palpebral commissures, and nasolabial folds. And, in T5, this variation occurred in the temporal points, medial palpebral, lateral palpebral, and labial commissures, and nasolabial folds.

Thus, the quantitative analysis showed that the patient's temperature variations were more persistent in the analysis points located on the lower face, where she had reported feeling pain.

After ruling out the neuropathies based on the results, the patient was referred for a dental reassessment. She was diagnosed with odontogenic pain - i.e., an orofacial pain of inflammatory origin, located on the lower left dental arch. This confirmed the presence of the hyper-radiant point revealed in Figure 4. 


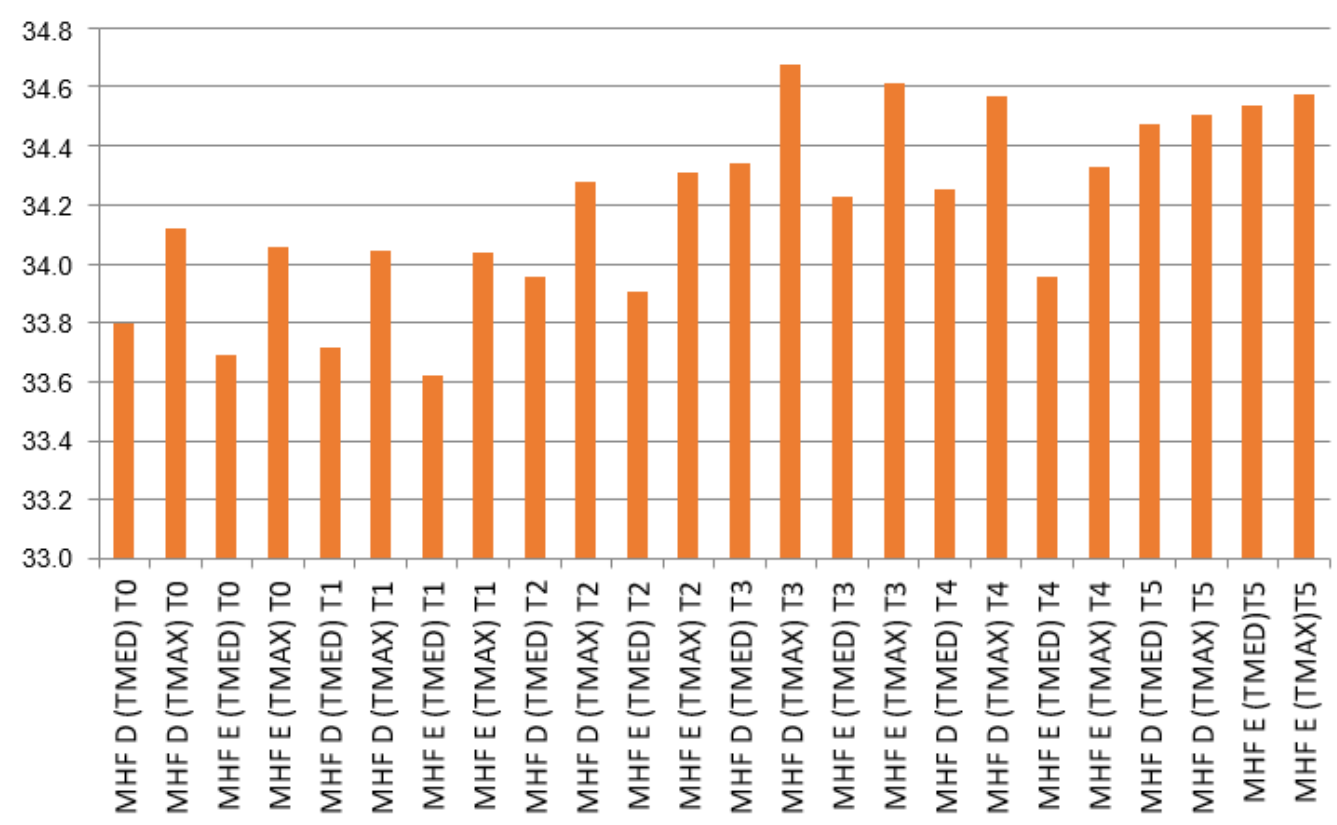

Captions: MRHF - mean of the right hemiface; MLHF - mean of the left hemiface; T0 = acclimatization; T1, T2, T3, T4, T5 = testing time in minutes; MAX T = maximum temperature; MEAN T = mean temperature.

Figure 5. Mean of the temperatures $\left({ }^{\circ} \mathrm{C}\right)$ in the right and left hemiface from T0 (acclimatization) to T5 (fifth minute of the cold stress test)

\section{DISCUSSION}

The human body's thermoregulation is controlled by the neurovegetative nervous system and can be described in two ways. The first of them refers to the information obtained from the peripheral receivers of the afferent sensitive nerves located in the skin. This information is sent to the upper pathways of the neurovegetative system, especially in the brainstem and upper cortical regions. Then, it is retransmitted to the hypothalamus, and the response is developed and sent via the efferent motor fibers to the smooth muscles of the vessels connected to the skin $^{13}$.

The second way refers to a response of the brainstem to a somatocutaneous or viscerocutaneous reflex. Impulses generated in glands and tissues such as muscles, bones, and joints can cause functional changes in the skin regarding temperature, sweating, tone, and sensitivity. Visceral and somatic diseases may influence the metabolism, temperature, and other skin properties through this arc reflex ${ }^{13}$.

According to what has been proposed in the literature concerning the human body thermoregulation, it was found that the patient's neurovegetative nervous system was normal. Hence, there was no small-fiber neuropathy or inflammatory neuropathy. During the cold stress test, the temperature changes followed a functional pattern - i.e., an end cooled down and others warmed up to preserve and maintain body homeostasis.

Moreover, a functional temperature change was identified in the patient's skin, which resulted from an inflammation caused by her dental problem. As further described in the literature, these visceral and somatic changes can influence both temperature changes and sensitivity, which happened to the patient. A hyperradiant point was identified in the lower left hemiface, revealing an increase in the local temperature. The patient had also reported episodes of pain and increased sensitivity in that site.

It is also important to point out that the central neurovegetative nervous system controls with the hypothalamus the skin blood flow to keep it uniform and symmetrical, thus maintaining normal thermoregulation. This results in a thermal pattern on both sides of the body, with symmetrical right and left sides. Hence, when the normal status is altered, qualitative and quantitative changes appear in the thermographic images $^{14}$.

According to what has been proposed in the literature and after analyzing the patient's thermographic images, it was observed that the images of the hands during the cold stress test had a symmetrical pattern, indicating that the central neurovegetative nervous system was normal. Also, the other thermographic images had asymmetries in some points - such as the 
cervical and lower left hemiface images - that were observed and qualitatively analyzed.

According to Haddad ${ }^{11}$, the central neurovegetative nervous system controls the skin blood flow to keep it uniform and symmetrical, which results in a symmetrical right and left thermal pattern. Thus, the qualitative and quantitative changes in thermal distribution (i.e., asymmetrical images) revealed an abnormal pattern in the thermographic images ${ }^{15}$.

The asymmetry indicated myofascial changes, agreeing with the patient's report of pain and dental clenching. According to Haddad ${ }^{12}$, the diagnosis of neurological and musculoskeletal changes confirmed with thermography is based on the local thermal imbalance between the muscles on the opposite sides. Thus, this temperature asymmetry is concordant with the presence of temporomandibular disorder ${ }^{15}$.

Haddad $^{12}$ established in her research the thermoanatomical points of the face and the maximum, mean, and minimum reference temperatures for thermographic analysis. She proposed 28 thermo-anatomical points -14 in the frontal view, seven in the right lateral view, and seven in the left lateral view. Moreover, she demonstrated that the temperatures whose change exceed $0.3 \stackrel{\circ}{\circ}$ from what has been proposed as normal should be approached as an abnormal temperature change, showing its asymmetrical pattern. These asymmetrical patterns usually occur due to the presence of sympathetic changes, traumatic lesions, or vascular or inflammatory changes - which was the case of the patient treated in this study ${ }^{12}$.

Therefore, this case report and all its qualitative and quantitative findings corroborate the data proposed in the literature.

\section{CONCLUSION}

Thermography was used as an auxiliary instrument to diagnose orofacial pain, in this clinical report, proving to be efficient. Also, the thermography helped rule out changes originating in peripheral, small-fiber, and inflammatory neuropathies, aiding the patient's differential diagnosis. The thermography also helped identify the thermo-anatomical points, which in turn helped precisely and noninvasively investigate the possible causes of the orofacial pain reported by the patient, enabling the qualitative and quantitative analyses.

\section{REFERENCES}

1. Leeuw R. Dor orofacial: guia de avaliação, diagnóstico e tratamento. 4aa ed. Nova Odessa: Quintessence; 2010.

2. Côrte ACR, Hernandez AJ. Termografia médica infravermelha aplicada à medicina do esporte. Rev Bras Med Esporte. 2016;22(4):315-9.

3. Loeser JD. Tic douloureux and atypical facial pain. J Can Dent Assoc. 1985;51(12):917-23.

4. Schestatsky P. Definition, diagnosis and treatment of neuro- pathic pain. Rev HCPA. 2008;28(3):177-87.

5. Maciel RN, Brioschi ML, Haddad DS, Arita ES, Corrêa CF, Balbinot LF. Dor orofacial crônica: diagnóstico por termografia infravermelha. Ribeirão Preto: Tota; 2016.

6. Balbinot LF. Termografia computadorizada na identificação de Trigger Points miofasciais [thesis]. Florianópolis (SC): Universidade do Estado de Santa Catarina; 2006.

7. Martinez JE, Grassi DC, Marques LG. Analysis of the applicability of different pain questionnaires in three hospital settings: outpatient clinic, ward and emergency unit. Rev Bras Reumatol. 2011;51(4):304-8.

8. Cunha TM, Giublin ML, Andrade Filho ACC, Siqueira JTT. Enfim, o que é dor e quais são seus mecanismos? In: Siqueira JTTS, Henriques AA, Kraychete DC, editors. 100 perguntas chave em dor. São Paulo: Permanyer Brasil Publicações; 2014. p.1-8.

9. Heimbecher CT, Ulbricht L. Termografia aplicada ao fenômeno de Raynaud: artigo de revisão bibliométrica. Pan Am $\mathrm{J}$ Med Thermol. 2018;4:15-23.

10. AAT: American Academy of Thermology. Guidelines for neuromusculoskeletal infrared medical thermography and sympathetic skin response (SSR) studies. [homepage on the internet]; [accessed 2020 nov 4]. Available at: https://aathermology. org/organization-2/guidelines/guidelines-for-neuromusculoskeletal-thermography/

11. Haddad DS. Correlação clínica e termográfica do ponto-gatilho miofascial nos músculos da mastigação [thesis]. São Paulo (SP): Universidade de São Paulo; 2011.

12. Haddad DS. Estudo da distribuição térmica da superfície cutânea facial por meio de termografia infravermelha: termoanatomia da face [dissertation]. São Paulo (SP): Universidade de São Paulo; 2014. 
13. Brioschi ML, Teixeira MJ, Silva MF. Princípios e indicações da termografia médica. São Paulo: Andreoli; 2010.

14. Uematsu S. Thermographic imaging of cutaneous sensory segment in patients with peripheral nerve injury. Skin temperature stability between sides of the body. J Neurosurg. 1985;62(5):716-20.

15. Weber M. Dor muscular e temperatura muscular [dissertation]. São Paulo (SP): Universidade de São Paulo; 2016. 